\title{
Erratum to: Key Intervention Characteristics in e-Health: Steps Towards Standardized Communication
}

\author{
Bridgette M. Bewick $^{1}$ - Steven J. Ondersma ${ }^{2}$. Mette T. Høybye ${ }^{3,4} \cdot$ Oskar Blakstad $^{5}$. \\ Matthijs Blankers ${ }^{6,7,8}$ • Håvar Brendryen ${ }^{9}$ Pål F. Helland ${ }^{10}$ • Ayna B. Johansen ${ }^{9}$. \\ Paul Wallace ${ }^{11}$ - Kristina Sinadinovic ${ }^{12}$. Christopher Sundström ${ }^{12}$. \\ Anne H Berman ${ }^{12,13}$
}

Published online: 6 July 2017

(C) International Society of Behavioral Medicine 2017

Erratum to: Int. J. Behav. Med.

DOI 10.1007/s12529-016-9630-3

In the original publication, Mette T. Høybye's and Christopher Sundström's names were misspelled as Mette T. Hoybye and Christopher Sundstrom. The errors have been corrected.

The online version of the original article can be found at http://dx.doi. org/10.1007/s12529-016-9630-3

Bridgette M. Bewick

b.m.bewick@leeds.ac.uk

1 University of Leeds, Leeds, UK

2 Merrill-Palmer Skillman Institute and Department of Psychiatry \& Behavioral Neurosciences, Wayne State University, Detroit, MI, UK

3 Interdisciplinary Research Unit, Elective Surgery Center, Regional Hospital Silkeborg, Silkeborg, Denmark

4 Department of Clinical Medicine, Aarhus University, Aarhus, Denmark

5 Explorable AS, Kristiansand, Norway

6 Trimbos Institute The Netherlands Institute of Mental Health and Addiction, Utrecht, The Netherlands
Arkin Mental Health Care, Amsterdam, The Netherlands

8 Department of Psychiatry, Academic Medical Centre, University of Amsterdam, Amsterdam, The Netherlands

9 The Norwegian Centre for Addiction Research, Institute of Clinical Medicine, Faculty of Medicine, University of Oslo, Oslo, Norway

10 Blue Cross Addiction Clinic, Oslo, Norway

11 Research Department of Primary Care and Population Health, University College London, London, UK

12 Department of Clinical Neuroscience, Center for Psychiatry Research, Karolinska Institutet, Stockholm, Sweden

13 Stockholm Center for Dependency Disorders, Stockholm, Sweden 